\title{
Multiple Ionization of Rare Gas Atoms Irradiated with Intense VUV Radiation
}

\author{
H. Wabnitz, ${ }^{1, *}$ A. R. B. de Castro, ${ }^{2,3}$ P. Gürtler, ${ }^{1}$ T. Laarmann, ${ }^{1, *}$ W. Laasch, ${ }^{1}$ J. Schulz, ${ }^{1, \ddagger}$ and T. Möller ${ }^{1, \S}$ \\ ${ }^{1}$ Hamburger Synchrotronstrahlungslabor HASYLAB at Deutsches Elektronen Synchrotron DESY, \\ Notkestrasse 85, 22607 Hamburg, Germany \\ ${ }^{2}$ LNLS, 13084-971 Campinas, São Paulo, Brazil \\ ${ }^{3}$ IFGW-UNICAMP, 13083-970 Campinas, São Paulo, Brazil \\ (Received 29 January 2004; published 18 January 2005)
}

\begin{abstract}
The interaction of intense vacuum-ultraviolet radiation from a free-electron laser with rare gas atoms is investigated. The ionization products of xenon and argon atomic beams are analyzed with time-of-flight mass spectroscopy. At $98 \mathrm{~nm}$ wavelength and $\sim 10^{13} \mathrm{~W} / \mathrm{cm}^{2}$ multiple charged ions up to $\mathrm{Xe}^{6+}\left(\mathrm{Ar}^{4+}\right)$ are detected. From the intensity dependence of multiple charged ion yields the mechanisms of multiphoton processes were derived. In the range of $\sim 10^{12}-10^{13} \mathrm{~W} / \mathrm{cm}^{2}$ the ionization is attributed to sequential multiphoton processes. The production of multiple charged ions saturates at 5-30 times lower power densities than at 193 and $564 \mathrm{~nm}$ wavelength, respectively.
\end{abstract}

PACS numbers: $32.80 . \mathrm{Rm}, 42.65 .-\mathrm{k}$

The interaction of intense laser radiation with atoms has been a subject of ever growing interest for more than two decades. The basic ionization mechanisms have been studied in great detail both experimentally and theoretically since the 1980s [1]. In the infrared and visible spectral range the strong field interaction with atoms is dominated by field ionization processes. On the other hand, at short wavelengths in the vacuum-ultraviolet (VUV) and x-ray regimes it is expected that the atoms will be ionized by multiphoton ionization. However, important questions such as the wavelength and intensity dependence of multiphoton processes and the scaling laws for cross sections at short wavelengths (VUV $\rightarrow$ $\mathrm{x}$ ray) remain open. Until now, experiments were hindered by the lack of sufficiently intense short wavelength light sources. This situation is presently changing. New and very intense sources like powerful high harmonic generation sources and free-electron lasers (FEL) are planned and under construction. In line with the development of new light sources numerous theoretical studies have been performed and have predicted various nonlinear processes in atoms [2-10]. Depending on the wavelength, nonlinear processes become important at rather high power densities of $10^{14}-10^{18} \mathrm{~W} / \mathrm{cm}^{2}$. Since all interaction of radiation starts on an atomic level, the understanding of the basic absorption and ionization processes is of great importance for all other future applications of short wavelength sources. The high intensity obtained recently at the VUVFEL at DESY [11] allows getting first results in the VUV. The findings can serve as a starting point for detailed comparison between experimental and theoretical results.

The VUV-FEL at DESY provides very intense VUV pulses between $80-100 \mathrm{~nm}$ of $30-100$ fs length. By focusing an intensity up to a few $10^{13} \mathrm{~W} / \mathrm{cm}^{2}$ can be achieved, which opens the door to strong field matter interactions. In strong field interactions two different regimes are considered for the ionization process: (a) photoabsorption by multiphoton processes and (b) tunneling when the electric field is strong enough for sufficiently bending the Coulomb barrier. These two regimes are separated by the Keldysh parameter $\gamma$, which is the ratio of the field frequency to the tunneling rate [12]. $\gamma$ separates the tunnel ionization regime $(\gamma \ll 1)$ from the multiphoton regime $(\gamma \gg 1)$. In the VUV $\gamma$ is much larger than 1 because of the high frequency of the radiation, and multiphoton ionization (MPI) can be expected to be the dominant ionization process in FEL experiments.

In this Letter we present experimental results of the interaction of intense VUV radiation with $\mathrm{Xe}$ and $\mathrm{Ar}$ atomic beams. The wavelength of the radiation was set to $98 \mathrm{~nm}$. Thus the energy of a single photon $(\sim 12.7 \mathrm{eV})$ is large enough to ionize neutral xenon atoms $\left(I_{p}=\right.$ $12.1 \mathrm{eV}$ ), but is below the first ionization potential of argon atoms at $15.76 \mathrm{eV}$ and $\mathrm{Xe}^{+}$at $21.2 \mathrm{eV}$. Accordingly, already the ionization of neutral argon (singly charged xenon) requires the (nonlinear) absorption of two FEL photons. The atomic beams were produced by expanding xenon (argon) gas at low stagnation pressure (10100 mbar) through a small nozzle into a vacuum that is connected to the experimental chamber by a skimmer. FEL radiation with a pulse duration of $\sim 100 \mathrm{fs}$ (FWHM) and pulse energies from $0.3-6 \mu \mathrm{J}$ was focused on the atomic beam using an elliptical mirror at grazing incidence. The details of the determination of the pulse length are given in [13]. By measuring the focal spot on a fluorescent screen with a charge-coupled device camera (maximum magnification 200), a diameter of $\sim 20 \pm 4 \mu \mathrm{m}$ was determined, resulting in a peak intensity up to $P_{\mathrm{FEL}} \sim 10^{13} \mathrm{~W} / \mathrm{cm}^{2}$. Ions produced in the interaction region were detected with a time-of-flight (TOF) mass spectrometer equipped with a multichannel plate (MCP) [14]. In the present work a TOF mass spectrometer with $150 \mathrm{~mm}$ length of the flight tube was used. The analog output signal of the MCP was read out by a digitizing oscilloscope. The pulse energy and time-of-flight mass spectra are recorded simultaneously on a pulse-to-pulse basis. Spectra taken at the same inten- 
sity $( \pm 2.5 \%)$ are averaged. Mass spectra of the ionization products of Xe and Ar atoms are shown in Figs. 1 and 2 for three different peak intensities of the FEL. The signal from $\mathrm{Xe}^{+}$is already saturated at $<10^{12} \mathrm{~W} / \mathrm{cm}^{2}$. This is expected when the large single photon absorption cross section of $\sim 40$ Mbarn [15] is taken into account. In the bottom trace in Fig. 1 the atomic ions $\mathrm{Xe}^{+}-\mathrm{Xe}^{3+}$ can be seen. Whereas $\mathrm{Xe}^{2+}$ and $\mathrm{Xe}^{3+}$ show a splitting due to different $\mathrm{Xe}$ isotopes, the shape of $\mathrm{Xe}^{+}$is unresolved, which indicates saturation of the MCP detector. Increasing the FEL peak intensity leads to the same saturation behavior for $\mathrm{Xe}^{2+}$. Simultaneously, the ion signal of $\mathrm{Xe}^{3+}$ increases strongly and higher charge states arise. At $\sim 1.3 \times 10^{13} \mathrm{~W} / \mathrm{cm}^{2}$ all charge states up to $\mathrm{Xe}^{6+}$ are present. The detection of multiple charged ions after irradiation of an atomic Xe beam is in contrast to mass spectra presented in a recent study of clusters [16]. In this study multiple ionization of Xe clusters is reported, and for comparison results of an atomic beam are presented. Two facts may account for the different findings: (i) experimental spectra presented in [16] and in this Letter were taken in two different experimental runs with slightly different operation settings of the FEL. In the first experimental run the FEL pulse duration was $50 \mathrm{fs}$ and in the second the length was about 100 fs. (ii) The data presented here were recorded with a new TOF mass spectrometer which has a higher sensitivity than the one used in [16]. The great sensitivity is due to a very sensitive bipolar MCP (BURLE) and a larger entrance aperture $(2 \mathrm{~mm})$ in the spectrometer. The larger aperture ensures that particles in a wide range of kinetic energies - especially particles with low energy or at rest-are detected very efficiently. We point out that the experimental results are reproducible within each of the two separate experimental runs and have been measured several times.

The mass spectra of Ar atoms in Fig. 2 look very similar. The corresponding charge states appear at somewhat

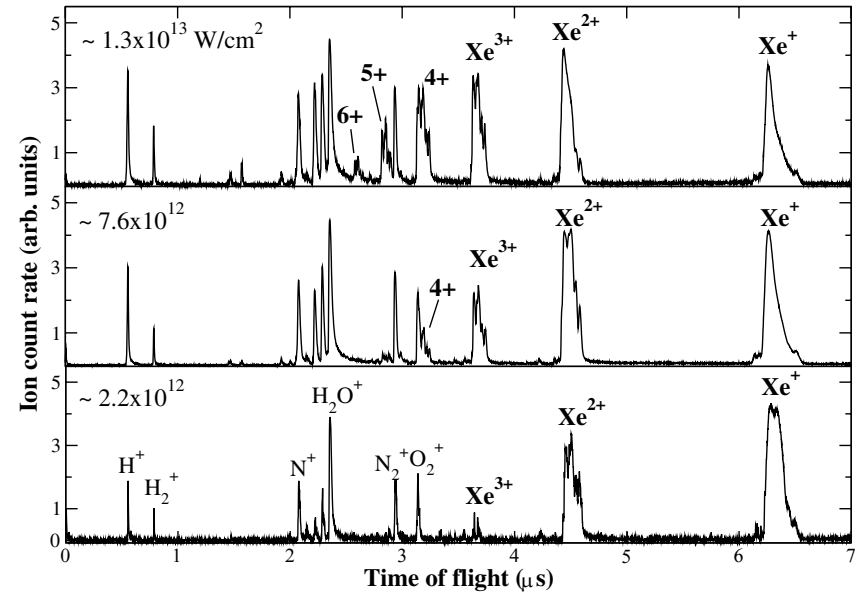

FIG. 1. Ionic products after irradiation of an atomic xenon beam for three different intensities of the FEL. Charge state and count rate of atomic ions strongly depend on $P_{\mathrm{FEL}}$. higher peak power densities. At $\sim 2 \times 10^{12} \mathrm{~W} / \mathrm{cm}^{2}, \mathrm{Ar}^{3+}$ is not yet detected, and at $\sim 2 \times 10^{13} \mathrm{~W} / \mathrm{cm}^{2}$ the ion signal of $\mathrm{Ar}^{4+}$ is very low. The mass spectra of both elements exhibit a strong dependence on the FEL peak intensity. This behavior points clearly to the nonlinear nature of the interaction with the intense FEL radiation at $98 \mathrm{~nm}$. In the following the underlying processes are addressed.

MPI is the multiphoton transition from a bound state to a free (continuum) state [17]. In lowest-order perturbation theory the $n$-photon ionization rate is given by

$$
\Gamma_{n}=\sigma_{n} P^{n},
$$

where $n$ is the minimum number of photons needed for ionization, $\sigma_{n}$ is the generalized cross section, and $P$ is the incident peak intensity.

In principle two different processes of MPI can be distinguished [1]. In the sequential ionization process electrons are detached one after another. For example, the production of an ion $A^{2+}$ can be described by the reactions

$$
\begin{gathered}
A+n_{(0,1)} \hbar \omega \rightarrow A^{+}+e^{-}, \\
A^{+}+n_{(1,2)} \hbar \omega \rightarrow A^{2+}+e^{-},
\end{gathered}
$$

where $n_{(0,1)}$ and $n_{(1,2)}$ denote the number of photons needed for the respective transitions. According to Eq. (1), the ion signal of $A^{+}$should follow in a $\log \Gamma-\log P$ diagram a straight line with the slope $n_{(0,1)}$ up to the saturation intensity $P_{s}$ and then remain almost constant. However, in the present experiments two additional effects cause a deviation from this behavior.

(i) $\mathrm{A}^{+}$ions originate not only from the interaction region of maximum intensity $P_{\max }$, but also from the wings of the spatial laser profile where $P<P_{\max }$. This effect causes a further increase in the ion signal above $P_{s}$. The slope of this increase is smaller than $n_{(0,1)}$, thus providing an indication of the value of $P_{s}$ [18].

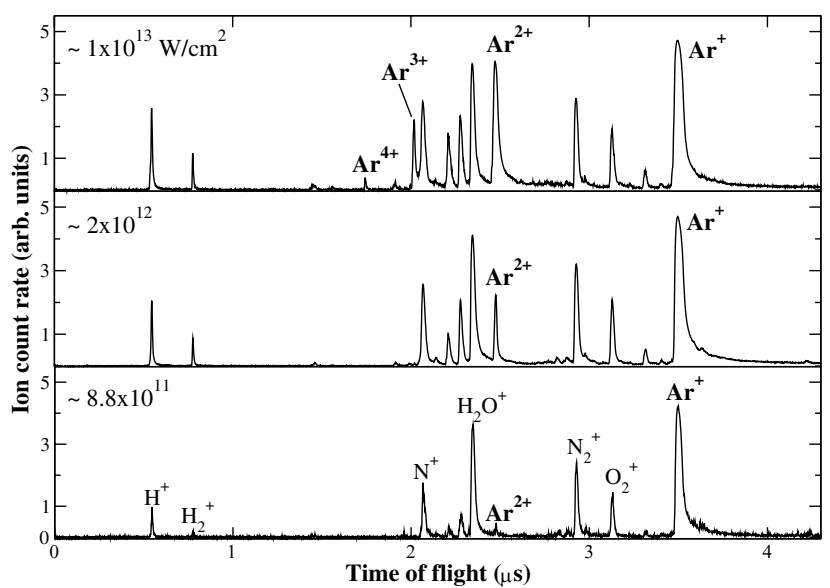

FIG. 2. Irradiation of an atomic argon beam at three different intensities $P_{\mathrm{FEL}}$. Compared with $\mathrm{Xe}$ atoms, equivalent charge states appear at higher $P_{\mathrm{FEL}}$. 
(ii) As the intensity increases the ions $A^{+}$are eventually further ionized and eject a second electron. This effect happens even before $P_{s}$ is reached and decreases the ionization count rate of $A^{+}$. But in most experiments the first effect leads to a small overall increase [18].

In direct MPI $A^{2+}$ is produced by a simultaneous detachment of two electrons.

$$
A+n_{(0,2)} \hbar \omega \rightarrow A^{2+}+2 e^{-},
$$

where $n_{(0,2)}$ photons $\left(n_{(0,2)} \leq n_{(0,1)}+n_{(1,2)}\right)$ are needed for the direct transition.

Recalling Eq. (1) we note that the nonlinearity of the MPI process can be determined by analyzing the slope of $\Gamma_{n}(P)$ in a log-log diagram. This is done in Fig. 3 for five different ion species. In Fig. 3 ionization rates of $\mathrm{Xe}^{3+}$ $\mathrm{Xe}^{5+}, \mathrm{Ar}^{2+}$ and $\mathrm{Ar}^{3+}$ are plotted as a function of FEL peak intensity. Straight lines indicate linear fits [19]. The deviation of $\mathrm{Xe}^{3+}$ and $\mathrm{Xe}^{4+}$ from the linear fits at higher peak power densities can be attributed to the saturation effect that all parent ions, $\mathrm{Xe}^{2+}$ and $\mathrm{Xe}^{3+}$, respectively, are ionized.

The saturation of $\mathrm{Xe}^{3+}$ coincides with the appearance of $\mathrm{Xe}^{4+}$, which is a first indication of sequential MPI [18]. However, the occurrence of saturation effects makes it difficult to model the ionization rates. In principle, the number of ions in a charge state $z$ due to sequential MPI can be expressed by

$$
N_{z}(P)=N_{z-1}(P) W_{(z-1, z)}(P)\left(1-W_{(z, z+1)}\right) .
$$

Here $W_{(z-1, z)}=\sigma_{(z-1, z)} P^{n_{(z-1, z)}} t_{l}$ is the ionization probability from $z-1$ to $z$ during the laser pulse duration $t_{l}, n_{(z-1, z)}$ is the minimum number of photons required for ionization from $z-1$ to $z$, and $\sigma_{(z-1, z)}$ is the corresponding
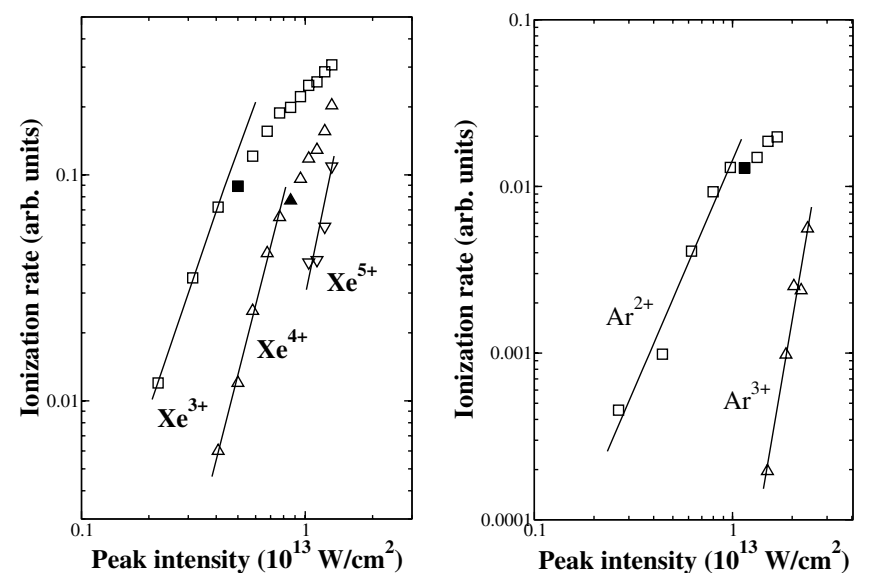

FIG. 3. Ion signal of $\mathrm{Xe}$ and Ar atoms as a function of peak intensity of the FEL at $98 \mathrm{~nm}$. Left: Ionization count rate of $\mathrm{Xe}^{3+}, \mathrm{Xe}^{4+}$, and $\mathrm{Xe}^{5+}$. The slopes of the linear fits (solid lines) reveal the order of the multiphoton process. Filled symbols denote graphically estimated saturation intensity for $\mathrm{Xe}^{3+}$ and $\mathrm{Xe}^{4+}$, respectively. Right: Ionization count rates of $\mathrm{Ar}^{2+}$ and $\mathrm{Ar}^{3+}$. generalized cross section. $N_{z-1}=N_{z-2}(P) W_{(z-2, z-1)}(P) \times$ $\left(1-W_{(z-1, z)}\right)$ denotes the number of $(z-1)$-charged ions. At saturation of $N_{z-1}$ the ionization probability $W_{(z-2, z-1)}$ becomes $\sim 1$. Under the assumption that further ionization to $z+1$ can be neglected $\left(W_{(z, z+1)} \ll 1\right)$ before saturation of $N_{z}$ occurs, Eq. (5) becomes

$$
N_{z}=N_{z-1} \sigma_{(z-1, z)} P^{n_{(z-1, z)}} t_{l}
$$

This simplified model is compared to the experimental results in Table I. In the second column in Table I the theoretical slopes $n_{(0, z)}$ of direct MPI are given. Results from Eq. (6) for sequential MPI are listed in the fourth column. For $\mathrm{Xe}^{3+}-\mathrm{Xe}^{5+}$ the experimentally derived slopes of the linear fits agree well with the number of photons $n_{(z-1, z)}$ needed for sequential ionization. For example, it needs $32.1 \mathrm{eV}$ to detach the outermost electron from $\mathrm{Xe}^{2+}$. Therefore $\mathrm{Xe}^{2+}$ has to absorb three $12.65 \mathrm{eV}$ photons, and the reaction becomes $\mathrm{Xe}^{2+}+3 \hbar \omega_{\mathrm{FEL}} \rightarrow$ $\mathrm{Xe}^{3+}+e^{-}$. This is in good agreement with the value of $2.9 \pm 0.2$ derived from the linear fit. On the other hand, direct MPI can be ruled out, because the neutral atom has to absorb six FEL photons for a simultaneous detachment of three electrons. The analysis of the $\mathrm{Xe}^{5+}$ reveals a slope well in the limits of a sequential process too. However, it has to be mentioned that the available intensity interval is much more limited than it is for the lower ion species $\mathrm{Xe}^{3+}$ and $\mathrm{Xe}^{4+}$. In the case of $\mathrm{Ar}^{2+}$, sequential MPI is also identified as the ionization process. The saturation of $\mathrm{Ar}^{2+}$ at $\sim 1.2 \times 10^{13} \mathrm{~W} / \mathrm{cm}^{2}$ coincides with the appearance of $\mathrm{Ar}^{3+}$. Analogous to $\mathrm{Xe}^{5+}$, there is only a narrow interval available to interpret the $\mathrm{Ar}^{3+}$ yield as a function of intensity. If one applies the analysis described above, linear fitting results in a slope of $6.9 \pm 1.9$ if an error bar of 0.07 for the pulse intensity is taken into account. The slope is closer to that of a direct process, but in view of the large error bar we cannot make a definitive statement whether in

TABLE I. Comparison of measured and theoretical nonlinearities of MPI process and saturation power densities $P_{s} . I_{p}$ denotes the binding energies of ion charge state $z$ when ionized from neutral atom (direct) or parent ion $(z-1)$ (sequential). $n_{(0, z)}$ and $n_{(z-1, z)}$ give the theoretical nonlinearity of the respective MPI process. The measured values of $n$ are given by the slopes and standard errors of the linear fits in Fig. 3. Quoted values of $P_{s}$ take systematic errors into account; see text.

\begin{tabular}{lrrrccc}
\hline \hline & \multicolumn{2}{c}{ Direct } & \multicolumn{2}{c}{ Sequential } & Measured & $P_{s}$ \\
& $I_{p}(\mathrm{eV})$ & $n_{(0, z)}$ & $I_{p}(\mathrm{eV})$ & $n_{(z-1, z)}$ & $n$ & $10^{13} \mathrm{~W} / \mathrm{cm}^{2}$ \\
\hline $\mathrm{Xe}^{2+}$ & 32.1 & 3 & 21.0 & 2 & $\cdots$ & $\cdots$ \\
$\mathrm{Xe}^{3+}$ & 65.2 & 6 & 32.1 & 3 & $2.9 \pm 0.2$ & $0.5 \pm 0.3$ \\
$\mathrm{Xe}^{4+}$ & 111.9 & 9 & 46.7 & 4 & $3.9 \pm 0.2$ & $0.9 \pm 0.6$ \\
$\mathrm{Xe}^{5+}$ & 171.6 & 14 & 59.7 & 5 & $4.1 \pm 1.2$ & $\cdots$ \\
$\mathrm{Ar}^{2+}$ & 43.4 & 4 & 27.6 & 3 & $2.8 \pm 0.6$ & $1 \pm 0.6$ \\
$\mathrm{Ar}^{3+}$ & 84.1 & 7 & 40.3 & 4 & $6.9 \pm 1.9$ & $\cdots$ \\
\hline \hline
\end{tabular}


the special case of $\mathrm{Ar}^{3+}$ it is a direct or a sequential process.

The experimental findings can be compared with experiments and theoretical work at longer wavelengths, especially $193 \mathrm{~nm}$. Mass spectra in [20] recorded with $193 \mathrm{~nm}\left(\sim 10^{14} \mathrm{~W} / \mathrm{cm}^{2}\right)$ show the same maximum ionization $\left(\mathrm{Xe}^{6+}\right)$ and the relative abundances of the charge states are about the same as in our work. Theoretical work for $193 \mathrm{~nm} \mathrm{[18]} \mathrm{comes} \mathrm{to} \mathrm{the} \mathrm{conclusion} \mathrm{that} \mathrm{the} \mathrm{satura-}$ tion of ionic charge states occurs at power densities of $5 \times 10^{13}-10^{14} \mathrm{~W} / \mathrm{cm}^{2}$. That is 5 to 10 times higher than the power densities needed at $98 \mathrm{~nm}$. For example, $P_{s}\left(\mathrm{Xe}^{3+}\right)$ is $\sim 5 \pm 3 \times 10^{12} \mathrm{~W} / \mathrm{cm}^{2}\left[P_{s}\left(\mathrm{Xe}^{4+}\right) \sim 9 \pm 6 \times\right.$ $\left.10^{12} \mathrm{~W} / \mathrm{cm}^{2}\right]$ in the FEL experiment as compared to $P_{s}\left(\mathrm{Xe}^{3+}\right) \sim 5 \times 10^{13} \mathrm{~W} / \mathrm{cm}^{2}$ at $193 \mathrm{~nm}\left[P_{s}\left(\mathrm{Xe}^{4+}\right) \sim 7 \times\right.$ $10^{13} \mathrm{~W} / \mathrm{cm}^{2}$, Fig. 4(c) in [18] ]. Further, it is interesting to compare our results with processes with the same total number of absorbed photons. According to [18] at $193 \mathrm{~nm}$ $\mathrm{Xe}^{2+}$ is produced by the sequential absorption of six photons, and its production saturates at $\sim 3.5 \times 10^{13} \mathrm{~W} / \mathrm{cm}^{2}$. At FEL photon energies the absorption of six photons is sufficient for producing $\mathrm{Xe}^{3+}$. As stated above, we measured that the ionization rate of $\mathrm{Xe}^{3+}$ saturates at $P_{s}\left(\mathrm{Xe}^{3+}\right) \sim 5 \times 10^{12} \mathrm{~W} / \mathrm{cm}^{2}$, which is once again considerably smaller than at $193 \mathrm{~nm}$.

Finally, our findings can be compared with early work on strong field ionization in the optical regime at $564 \mathrm{~nm}$ [21]. The saturation intensities for $\mathrm{Xe}^{+}-\mathrm{Xe}^{5+}$ are even higher than at $193 \mathrm{~nm}$. For $\mathrm{Xe}^{3+}$ the saturation intensity is $\sim 2 \times 10^{14} \mathrm{~W} / \mathrm{cm}^{2}$, which is 40 times higher than at $98 \mathrm{~nm}$ and 4 times higher than at $193 \mathrm{~nm}$. The clear trend that the saturation intensities decrease with increasing laser frequency is supported by recent theoretical work [22].

In summary, we report first results on the ionization of rare gas atoms with intense VUV pulses. Multicharged ions are created by sequential MPI. The saturation intensities are up to 1 order of magnitude lower than that obtained with $193 \mathrm{~nm}$ wavelength radiation. The results provide first data on multiphoton processes in the VUV. They can serve as a starting point for a joint effort of experiment and theory to obtain a detailed understanding of multiphoton processes at short wavelengths.

We thank A. Swiderski for technical support in the design and construction of the cluster experiment and the TTF team at DESY for providing the FEL beam and the photon diagnostics. One of the authors (H.W.) is grateful to Peter Lambropoulos for helpful comments. In addition, we thank R. Santra for making calculations available prior to publication.

*Present address: CEA-SPAM, Centre d'Etudes de Saclay, 91191 Gif-sur-Yvette, France.

Electronic address: wabnitz@drecam.cea.fr

†Present address: Max Born Institute, Max-Born-Strasse 2a, 12489 Berlin, Germany.

*Present address: MAX-lab, Box 118, 22100 Lund, Sweden.

${ }^{\S}$ Present address: Technische Universität Berlin, Hardenbergstrasse 36, 10623 Berlin, Germany.

[1] N. Delone and V. Krainov, Multiphoton Processes in Atoms (Springer, New York, 2000).

[2] A. Saenz et al., J. Phys. B 32, 5629 (1999).

[3] T. Nakajima et al., Europhys. Lett. 57, 25 (2002).

[4] U. Saalmann et al., Phys. Rev. Lett. 89, 143401 (2002).

[5] U. Lambrecht et al., Phys. Rev. A 57, 2832 (1998).

[6] J. Bauer et al., J. Phys. B 34, 2245 (2001).

[7] M. Brewczyk et al., J. Phys. B 34, L289 (2001).

[8] M. Brewczyk et al., J. Phys. B 32, L1 (1999).

[9] M. Kornberg et al., J. Synchrotron Radiat. 9, 298 (2002).

[10] S. Novikov et al., J. Phys. B 33, 2287 (2000).

[11] V. Ayvazyan et al., Phys. Rev. Lett. 88, 104802 (2002).

[12] L. Keldysh, Sov. Phys. JETP 20, 1307 (1965).

[13] V. Ayvazyan et al., Nucl. Instrum. Methods Phys. Res., Sect. A 507, 368 (2003).

[14] We note that comparing experimental data at reduced $P_{\mathrm{FEL}}$ by geometrical means and reduced pulse energy confirms the focal spot size measurement. A diameter of $20 \mu \mathrm{m}$ explains consistently experimental TOF spectra taken at different positions inside and outside the focal spot.

[15] M. Richter et al. (to be published).

[16] H. Wabnitz et al., Nature (London) 420, 482 (2002).

[17] M. Protopapas et al., Rep. Prog. Phys. 60, 389 (1997).

[18] P. Lambropoulos et al., J. Opt. Soc. Am. B 4, 821 (1987).

[19] Conservative estimates of $\pm 50 \%$ for the pulse duration and $\pm 20 \%$ for the focal spot size are taken into account to determine the error in $P_{\mathrm{FEL}}$. As these are systematic errors, they do not affect the slopes in Fig. 3. Thus no error bars are displayed in Fig. 3. Standard errors of the slopes resulting from the linear fitting routine are given in Table I. The saturation power densities for $\mathrm{Xe}^{3+}, \mathrm{Xe}^{4+}$ and $\mathrm{Ar}^{2+}$ include the systematic errors of $P_{\mathrm{FEL}}$.

[20] T. Luk et al., Phys. Rev. Lett. 51, 110 (1983).

[21] M. Perry et al., Phys. Rev. Lett. 60, 1270 (1988).

[22] R. Santra et al., Phys. Rev. A 70, 053401 (2004). 